\title{
Title:Eradicating metastatic cancer and the evolutionary dynamics of extinction
}

Authors: Robert Gatenby, $\mathrm{MD}^{1,2,3}$ and Joel Brown, $\mathrm{PhD}^{1,3}$

Affiliation: ${ }^{1}$ Cancer Biology and Evolution Program, ${ }^{2}$ Department of Diagnostic Imaging and Interventional Radiology, ${ }^{3}$ Department of Integrated Mathematical Oncology, Moffitt Cancer Center, Tampa, Florida 33612 USA

\section{Corresponding author:}

Robert Gatenby, MD Moffitt Cancer Center 12902 Magnolia Dr

Tampa, FL 33612

Phone: (813) 745-2843

Email: Robert.Gatenby@Moffitt.org

\section{Competing Interests}

None

\section{Author Contributions}

The authors contributed equally to the theoretical development, background investigations, and preparation of the manuscript.

\section{Key words}

Cancer treatment, resistance to treatment, evolution of resistance, background extinctions.

\section{Abstract}

We propose the traditional goal of cancer therapists to develop a single drug or drug combination that can, by itself, eliminate all cancer cells within a host has neglected potential treatments that may achieve curative outcomes by strategically combining agents that are individually effective but noncurative. We derive basic principles for such an approach from the eco-evolutionary dynamics of background extinctions in which a "first strike" reduces the size and heterogeneity of the initial population and is followed immediately by demographic and ecological "second strikes" that push the population below an extinction threshold. This proposed strategy appears identical to the empiricallyderived curative therapy in childhood Acute Lymphocytic Leukemia.

\section{Synopsis}


For decades, cancer therapists have focused on development of new drugs as the most productive strategy to improve outcomes in treatment of disseminated, metastatic cancers. The success and failure of these efforts is evident in, for example, the large number of drugs (about 52 (1)) approved for treatment of metastatic prostate cancers (mPC) and the grim reality that $\mathrm{mPC}$ remains uniformly fatal. Nevertheless, it is generally assumed that curative treatment for mPC and other common cancers will require newer, better drugs; and the search continues. Here we propose the main barrier to cure in many metastatic cancers including $\mathrm{mPC}$ is not insufficient agents but rather insufficient tactics.

Currently, first, second and third line treatments for metastatic cancers are viewed as independent events each having the identical goal of maximally killing of cancer cells with complete eradication and cure as the optimal outcome. Typically, drugs are applied at maximum dose density and, if the tumor initially decreases in size, continue to be administered until excessive toxicity or tumor progression mandates a change.

The similarity of cancer treatment to extinction dynamics has been previously noted. In this context, conventional therapy mimics the famous mass extinction of the dinosaurs by a single catastrophic event - the worldwide effects caused by the K-T impact. Maximum dose density treatments represent similar application of immense evolutionary force which, by itself, will ideally cause extinction of the cancer population.

Here we propose that mass extinction is a poor paradigm for cancer therapy because the indiscriminate effects of massive global perturbations constrain its clinical application where collateral damage to normal cells can be lethal to the host. Furthermore, since cancer cells need only to be more resistant to the treatment than the host cells, surviving cancer subpopulations are virtually inevitable.

We present an alternative strategy based not on the dynamics of the catastrophic K-T impact but rather on the sequence of eco-evolutionary events typically observed in background extinctions. Decline of a large, diverse, and geographically dispersed species generally begins with one or more 
demographic and ecological perturbations. This "first strike" reduces the size of the population and produces an evolutionary bottleneck with a small surviving cohort, limited by low genetic diversity and a fragmented ecology, rendered highly vulnerable to small, stochastic ecological and demographic events that would not have significantly affected the population prior to the first strike.

Thus, we hypothesize large, diverse, and spatially-dispersed metastatic cancers may be eradicated by a strategic application of a sequence of drugs or drug combinations none of which are, individually, curative. The specific sequence, similar to the dynamics of background extinctions, begins with a first strike to reduce the cancer population size and diversity followed immediately by several intense but brief eco-evolutionary perturbations to drive the vulnerable surviving population to its extinction threshold. We note these predicted dynamics are consistent with empirically-derived, curative therapy for Acute Lymphoblastic Leukemia (ALL) in which the initial "induction" treatment represents a first strike followed quickly by a second strike ("treatment intensification") followed by a third strike ("delayed intensification"), and a fourth strike ("maintenance"). We demonstrate a similar strategy could be applied in metastatic prostate cancer.

\section{Introduction}

In an evolutionary model, the cancer cell population within a host is a clade (i.e. descending from one cell or one population) but the heterogeneous intratumoral environment typically generates multiple ecological niches occupied by phenotypically and genetically distinct cancer cell "species" (2, 3). Cancer cells compete with each other in a dynamic environment with spatial and temporal fluctuations in nutrients as well as blood-borne growth factors while being "stalked" by the predatory cells of the host immune system. Adding to the eco-evolutionary complexity is the ability of the cancer cell to deploy niche-construction strategies such as angiogenesis (4) thus generating ecological as well as evolutionary heritability.

Up to a billion cancer cells (5) can occupy each gram of tumor and many metastatic human cancers reach a total tumor burden well in excess of 100 grams. Eliminating this large, diverse, and 
spatially dispersed population (roughly equivalent to the size and diversity of the global mouse population (6)), without destroying the native cellular species necessary for host survival, is clearly a daunting task. For over a century, cancer therapists have largely focused on drug development as the best means to achieve this goal. The ideal cancer drug is a "silver bullet" that eradicates all cancer cells and spares all normal ones (7). Unfortunately, even highly targeted therapies frequently cause significant toxicity to normal host cells so that silver bullets, equivalent to antibiotics in bacterial infections, remain elusive.

Thus, most cancer treatments represent a trade-off between the benefit of killing as many cancer cells as possible and the potentially lethal toxicity to normal cells necessary for host survival (8). Many agents using different mechanisms of action have been identified but all receive approval based on measurable reduction of tumor volume and increased host survival. The success and failure of these efforts are evident in treatments for metastatic prostate cancer (mPC). Currently, oncologists can select from about 50 drugs(1) approved for treating men diagnosed with mPC. Yet, of the estimated 37,000 men in the US who will develop mPC in the next year, none will be cured. Nevertheless, most therapist assume curative treatment in MPC and other metastatic cancers can be obtained only through development of new and better drugs by academic institutions and the international pharmaceutical industry.

Here we hypothesize that, in many cancers including $\mathrm{mPC}$, the limitations in treatment outcomes are not the absence of sufficiently effective drugs but rather the absence sufficiently effective tactics. We propose that a fundamental error in current treatment strategies is the expectation that cure must be obtained from one drug or combination of drugs administered continuously until all cancer cells are eradicated. Decades of experience in treatment of disseminated cancers has clearly demonstrated such an outcome, in large, heterogeneous, spatially dispersed, and rapidly evolving cancer populations, is unlikely and, in fact, may never be consistently attainable. However, we hypothesize that curative outcomes may be achievable through strategic sequencing of drugs or combinations of drugs each of which is effective but not curative. And, we propose the 
guiding principles for this alternative treatment approach are apparent in the evolutionary dynamics of species extinctions observed in nature.

\section{Cancer treatment and the evolutionary dynamics of extinction}

In prior work, we have used evolutionary dynamics to control metastatic cancers when cure was not clinically considered to be an achievable $(7,9-14)$. However, continued theoretical investigations of the eco-evolutionary dynamics of cancer treatment have suggested the historic oncologic focus on treatment agents may have neglected opportunities to obtain curative outcomes that are can be observed in extinction events (15).

Well over $99 \%$ of species that have arisen during earth's history, many of them more numerous and diverse than metastatic cancers, have become extinct and the analogy to cancer treatment has been previously noted $(16,17)$. A famous, but perhaps misleading, extinction is that of the dinosaurs following a catastrophic meteor impact. When cancer treatment applies toxic drugs at maximum tolerated dose (MTD), it mimics in many ways the powerful ecological forces that produce mass extinctions. But, decades of experience have demonstrated this approach is largely ineffective in producing the extinction of most metastatic cancers. Why? One obvious limitation, also apparent in mass extinctions (18), is that large perturbations are inherently indiscriminate and so the amplitude of cancer treatment is constrained by the danger of causing potentially fatal collateral damage to normal cells. Thus, on average, cancer cells can evade the toxicity of treatment if they are simply more resistant than normal cells necessary for host survival. Given the diversity of metastatic cancer populations and their substantial evolutionary capacity, resistant cells are highly likely to be present prior to therapy or emerge quickly upon therapy initiation. To address these limitations, the world-wide pharmaceutical industry continues to search for new drugs that deliver greater killing power and improved specificity. We note, however, that this effort in new drug development implicitly assumes that cancer eradication must be achieved by one definitive treatment using a single drug or combination of drugs similar to the dinosaur extinction by the K-T impact. 
There is however an alternative approach apparent in the less dramatic and more nuanced dynamics of background extinctions. Consider, for example, the heath hen (Tympanuchus cupido cupido), a large chicken-like bird that was populous on the east coast of North America when European settlers first arrived. Throughout the colonial period, the heath hen population steadily declined due to hunting (the heath hen may have been the "turkey" at the first Thanksgiving) and habitat disruption from expanding settlements (19). By 1870, just 50 heath hens remained; all restricted to a small refuge on the island of Martha's Vineyard. With protection from the local community, their population rebounded to about 2,000 by 1915 (20). However, the next few years brought stochastic perturbations. A fire destroyed part of their breeding area, several winters were unusually harsh, and an infectious poultry disease appeared. The last heath hen died in 1932.

The heath hen's decline from a large, spatially dispersed, heterogeneous population is a welldocumented, well-studied background extinction that illustrates two important ecological and evolutionary concepts (21): (1). The role of multiple sequential perturbations in extinction divided into "First Strike - Second strikes"(15) strategies. (2). The concept of Minimum Viable Population (MVP)(22).

The "first strike" is typically one or several events that greatly reduce the size, spatial distribution, and diversity of an initially large and heterogeneous population. For the heath hen, the first strike involved habitat loss and over-hunting. The events that produce the first strike are generally deterministic; their consequences are reasonably predictable and reproducible. The heath hen story is also typical in that the first strike did not eliminate the entire population. Rather, it caused an evolutionary bottleneck that within a small, isolated populations with limited genotypic and phenotypic diversity(23). The extinction was actually caused by small, unpredictable perturbations that would have been inconsequential to the original large, heterogeneous, geographically dispersed population. But. the heath hen was near its MVP because the small homogeneous and geographically isolated colony could neither recover from nor adapt to a series of relatively minor stochastic perturbations. 
The eco-evolutionary dynamics of extinction in species at or near their MVP have been extensively investigated in conservation studies using the framework of Population Viability Analysis (PVA)(24), which estimates the likelihood of extinction and identifies strategies to promote conservation. Many of the principles used for PVAs (25) potentially apply to small cancer populations though, ironically, with a goal of increasing the probability of extinction rather than the usual conservation goal of preventing it.

To begin, we note that some important components of PVA are not applicable to cancer populations. For example, cancer cells, because they reproduce asexually, do not need to locate mates. However, a number of other vulnerabilities may be critical in small cancer populations. For example, the surviving cells, when compared to the initial population, are likely more resistant to the agents of the first strike but less genetically heterogeneous. While the former renders them relatively invulnerable to the mechanisms that achieved the first strike, the latter may increase their susceptibility to different treatments. Furthermore, the evolutionary trade-offs that governed evolution of resistance may render the cells uniquely vulnerable to treatments that specifically targets the resistance mechanism(s).

These dynamics are probably observable clinically in adjuvant cancer therapy. For example, when pediatric patients with clinically localized osteosarcoma were treated with only surgical resection, about $80 \%$ developed lethal metastases within two years. However, if chemotherapy was administered after surgery, the development of metastases fell to as low as $10 \%(26)$. In an ecoevolutionary context, it is likely that clinically unobservable micro-metastases at or near their MVPs are present in nearly all osteosarcoma patients. Untreated, some of these micro-tumors undergo extinction while others stochastically expand to form a clinical tumor in about $80 \%$ of patients. Adjuvant treatment following the surgical first strike reduces the number of cancer cells in each metastatic site pushing them closer to and frequently below their MVP. This increases the probability of extinction and decreases the probability that the small cancer colonies will grow to clinically evident metastases. 


\section{Clinical application}

The evolutionary dynamics of extinctions suggest the greatest probability of eliminating a metastatic cancer population will occur through a strategic integration of multiple therapies. A first strike is administered not with a goal or eradicating the cancer population but reducing its size (Figures 2). This should be followed rapidly (i.e. before the resistant populations begin to proliferate) by second strikes which use different strategies to exploit the vulnerability of the survivors to drive the population below the absorbing, extinction boundary (Figure 3).

This approach would require two major changes in common oncologic practices. First, the treating physician will need to switch therapy despite the high level of efficacy in the first strike agents. Second, the physician needs to apply treatment even in the absence of visible tumor (i.e. "measurable disease") so that the effects of treatment cannot be assessed with current technology.

Can the evolutionary lessons from background extinctions guide treatment strategies in metastatic cancer? Importantly, there is a precedent for this in pediatric Acute Lymphoblastic Leukemia in which a highly successful, empirically-derived curative therapy has been developed through a number of clinical trials over several decades. Typically, pediatric ALL treatment begins applies wit an initial "induction" therapy (14) that is followed immediately by a "treatment intensification" using new agents and then by an "intermediate dose intensification" and then by "maintenance" also using different agents. In the context of background extinctions, the initial induction treatment represents a first strike followed quickly by a second strike ("treatment intensification"), third strike ("delayed intensification"), and fourth strike ("maintenance"). In effect, the optimal therapy predicted by the background extinction model and evidenced in the ALL treatment is to use the first strike to deliver substantially damage the tumor population and then simply continue to "kick them when they are down."

Can the dynamics of background extinction be adapted for the treatment of metastatic prostate cancer (mPC)? As noted above, ADT is usually an effective first strike that greatly reduces the cancer population's size and diversity (i.e. strongly selecting for "castrate resistant" phenotypes). 
Microscopy of surviving tumor populations following neoadjuvant ADT therapy for localized prostate cancer typically finds the ADT results in fragmented tumors with small clusters of cells "floating" in large regions of necrosis(27). Such isolated pockets are typically "decoupled"(28) because the cell populations may be too small and separated to promote mutually beneficial angiogenesis, and too distant to allow cell migration from one island to another. These small isolated colonies should be vulnerable to unpredictable habitat disruption (environmental stochasticity) caused by (for example) temporal variations in regional blood flow(29). Small cancer cell populations may also lose survivalenhancing aggregation (Allee(30)) (31) effects such as "safety in numbers" when subjected to the predatory activities of the immune system or "dilution effects" of multiple cellular "sinks" that can reduce the effective concentration of a treatment drug. Finally, small variations in the local proliferation and death of the cancer cells (demographic stochasticity) might, through a random walk process, cause sporadic decreases in populations toward a lower limit ("extinction boundary"(32)) at which extinction becomes inevitable.

These eco-evolutionary dynamics of background extinctions suggests that the probability of eradicating $\mathrm{mPC}$ can be substantially increased by a vigorous, multi-modal treatment of the small mPC cell populations that persist following an effective first strike with ADT. In effect, "kick them when they are down" even when the effects of treatment cannot be measured directly through imaging or serum biomarkers.

However, most mPC treatment protocols continue to administer ADT even after the PSA normalizes. Treatment changes only in the event of unacceptable toxicity or unambiguous evidence of tumor progression. This cannot be an optimal strategy for cure because the surviving cells have survived intense selection pressure and so must generally be resistant to ADT. Continuing therapy will not further reduce the population size and eventual tumor progression is inevitable. Furthermore, because therapy is changed only when the PSA increases, the new treatment is applied to a larger population with fewer extinction vulnerabilities than when the PSA was at its nadir. In other words, conventional therapy fails to "kick them when they are down." 
Ideally, extinction-producing therapies for metastatic prostate cancer will use treatments with mechanisms of action and resistance very different from the initial first strike with ADT. Fortunately, these drugs probably already exist. Importantly, optimal agents for this phase of therapy do not necessarily have to be effective as first strike drugs. That is, because the residual tumor populations are both small and relatively homogenous, as illustrated in the Heath Hen extinction, treatments not effective against large, diverse tumor populations may nevertheless be highly successful in reducing small homogeneous populations below their MVP. In mPC following ADT, for example, treatments to reduce the size of the resistant populations might include drugs that specifically target resistant mechanisms (e.g. abiraterone, a CYP17 inhibitor (33)) or cytotoxic chemotherapy drugs such as Docetaxel (34). Additional perturbations might include habitat disruption through angiogenesis inhibitors (35) or introduction of a "predator" through immunotherapy (36). Although neither approach is currently very effective when treating large volumes of metastatic prostate cancer $(37,38)$, they may be sufficiently effective can to push the small, homogeneous surviving population past the extinction boundary. Of course, additional empirical and theoretical investigations will be necessary to determine an optimal, potentially strategy in prostate cancers following the ADT first strike.

\section{Conclusion}

Theoretic studies play a critical role in science by questioning conventional wisdom and presenting alternatives hypotheses to stimulate discussion. Here, our theoretical model for curative outcomes in metastatic cancers builds upon multiple prior studies examining the evolutionary dynamics of cancer therapy $(9,14,39)$. Using lessons from background extinctions, we suggest that curative outcomes may be obtained using a strategic sequence of treatments each with different goals. The first strike is used to reduce the size and heterogeneity of a disseminated cancer population leaving behind a genetic bottleneck with a small, fragmented, relatively homogeneous population. Further treatment of this resistant population with the first strike agents is probably futile because the surviving population has likely been selected for resistance. Furthermore, declaring the tumor "in remission" and waiting for it to become clinically evident before initiating further treatment is 
allows the cancer population to recover. By the time it becomes clinically evident, the size and diversity of the cancer subpopulations likely precludes a successful second strike. Thus, as in treatment of ALL, the second strike must immediately follow the first strike and should use different agents. Furthermore, it is likely that these follow-up strikes can be brief but intensive since the goal is to apply as many perturbations as possible to push the surviving population below their extinction threshold. . Importantly, these treatments must be administered even in the absence of measurable disease based on historic observations that recurrence even after a highly first strike is inevitable.

Finally, this alternative strategy may require new thinking in drug development. Current drug approvals are generally based on their ability to reduce the size of large tumors. That is, they are only evaluated as first strike agents. However, drugs that fail to significantly alter the size of large heterogeneous tumors may nevertheless be extremely effective in reducing small tumor populations that persist following a first strike (Figures 1 and 2). Furthermore, ideal "kick them when they are down" drugs may be quite different from those usually envisioned for the particular type of cancer. Thus, optimal drug selection may need modified based on the clinical context for its use.

In summary, the traditional focus on generating new cancer drugs has successfully produced large numbers of treatment options for many cancers. However, silver bullets remain elusive for most metastatic diseases, and MTD therapies are limited by toxicity and the evolution of resistance. Lessons from observations of extinctions in Nature suggest optimal cancer treatment may be found in the eco-evolutionary dynamics of background extinctions so that strategic multi-move application of available drugs may be sufficient to eradicate some currently incurable metastatic cancers.

\section{Acknowledgements}

We thank Drs. John Cleveland, Damon Reed, Patrick O'Kane, and Robert Stern for their critical review of the Perspective. This work was supported by the European Union's Horizon 2020 research and innovation program (grant agreement No 690817), the James S. McDonnell Foundation grant, "Cancer therapy: Perturbing a complex adaptive system," a V Foundation grant, NIH/National Cancer Institute (NCl) R01CA170595, Application of Evolutionary Principles to Maintain Cancer Control (PQ21), and NIH/NCI U54CA143970-05 [Physical Science Oncology Network (PSON)] "Cancer as a complex adaptive system." 


\section{References}

1. www.drugs.com/condition/prostate-cancer.html. 2018 [

2. Ibrahim-Hashim A, Robertson-Tessi M, Enriquez-Navas PM, Damaghi M, Balagurunathan Y, Wojtkowiak JW, et al. Defining Cancer Subpopulations by Adaptive Strategies Rather Than Molecular Properties Provides Novel Insights into Intratumoral Evolution. Cancer Res. 2017;77(9):2242-54.

3. Gatenby RA. Population ecology issues in tumor growth. Cancer Res. 1991;51(10):2542-7.

4. Qian JJ, Akcay E. Competition and niche construction in a model of cancer metastasis. PLoS One. 2018;13(5):e0198163.

5. DeVita VT, Jr., Young RC, Canellos GP. Combination versus single agent chemotherapy: a review of the basis for selection of drug treatment of cancer. Cancer. 1975;35(1):98-110.

6. Harr B, Karakoc E, Neme R, Teschke M, Pfeifle C, Pezer Z, et al. Genomic resources for wild populations of the house mouse, Mus musculus and its close relative Mus spretus. Sci Data. 2016;3:160075.

7. Gatenby RA. A change of strategy in the war on cancer. Nature. 2009;459(7246):508-9.

8. Norton L, Simon R. The Norton-Simon hypothesis revisited. Cancer Treat Rep. 1986;70(1):163-9.

9. Zhang J, Cunningham JJ, Brown JS, Gatenby RA. Integrating evolutionary dynamics into treatment of metastatic castrate-resistant prostate cancer. Nat Commun. 2017;8(1):1816.

10. Enriquez-Navas PM, Kam Y, Das T, Hassan S, Silva A, Foroutan P, et al. Exploiting evolutionary principles to prolong tumor control in preclinical models of breast cancer. Sci Transl Med. 2016;8(327):327ra24.

11. Cunningham JJ, Gatenby RA, Brown JS. Evolutionary dynamics in cancer therapy. Mol Pharm. 2011;8(6):2094-

100.

12. Gatenby R. Perspective: Finding cancer's first principles. Nature. 2012;491(7425):S55.

13. Gatenby R, Brown J. The Evolution and Ecology of Resistance in Cancer Therapy. Cold Spring Harb Perspect Med. 2018;8(3).

14. Gatenby RA, Silva AS, Gillies RJ, Frieden BR. Adaptive therapy. Cancer Res. 2009;69(11):4894-903.

15. Raup DM. Extinction : bad genes or bad luck? New York: W.W. Norton; 1991. xvii, 210 p. p.

16. Walther V, Hiley CT, Shibata D, Swanton C, Turner PE, Maley CC. Can oncology recapitulate paleontology? Lessons from species extinctions. Nat Rev Clin Oncol. 2015;12(5):273-85.

17. Korolev KS, Xavier JB, Gore J. Turning ecology and evolution against cancer. Nat Rev Cancer. 2014;14(5):371-80.

18. Hull P. Life in the Aftermath of Mass Extinctions. Curr Biol. 2015;25(19):R941-52.

19. Pannell JH. The Heath Hen. Science. 1943;98(2538):174.

20. Headstrom BR. Preservation of the Heath Hen. Science. 1928;68(1749):15.

21. Brook BW, Alroy J. Pattern, process, inference and prediction in extinction biology. Biol Lett. 2017;13(1).

22. Brook BW, Bradshaw CJ, Traill LW, Frankham R. Minimum viable population size: not magic, but necessary. Trends Ecol Evol. 2011;26(12):619-20; author reply 20-2.

23. Johnson JA, Dunn PO. Low genetic variation in the heath hen prior to extinction and implications for the conservation of prairie-chicken populations. Conserv Genet. 2006;7(1):37-48.

24. Boyce MS. Population Viability Analysis. Annu Rev EcolSyst. 1992;23:481-506.

25. Beissinger SR, McCullough DR. Population viability analysis. Chicago: University of Chicago Press; 2002. xvi, 577

p. p.

26. Perkins SM, Shinohara ET, DeWees T, Frangoul H. Outcome for children with metastatic solid tumors over the last four decades. PLoS One. 2014;9(7):e100396.

27. Armas OA, Aprikian AG, Melamed J, Cordon-Cardo C, Cohen DW, Erlandson R, et al. Clinical and pathobiological effects of neoadjuvant total androgen ablation therapy on clinically localized prostatic adenocarcinoma. Am J Surg Pathol. 1994;18(10):979-91.

28. Channell RL, M.V. Trajectories to extinction: spatial dynamics of the contraction of geographical ranges. Journal of Biogeography. 2001;27(1):169-79.

29. Gillies RJ, Brown JS, Anderson ARA, Gatenby RA. Eco-evolutionary causes and consequences of temporal changes in intratumoural blood flow. Nat Rev Cancer. 2018;18(9):576-85.

30. Konstorum A, Hillen T, Lowengrub J. Feedback Regulation in a Cancer Stem Cell Model can Cause an Allee Effect. Bull Math Biol. 2016;78(4):754-85.

31. Brown JS, Cunningham JJ, Gatenby RA. Aggregation Effects and Population-Based Dynamics as a Source of Therapy Resistance in Cancer. IEEE Trans Biomed Eng. 2017;64(3):512-8. 
32. Boukal DS, Berec L. Single-species models of the Allee effect: extinction boundaries, sex ratios and mate encounters. J Theor Biol. 2002;218(3):375-94.

33. Fizazi K, Tran N, Fein L, Matsubara N, Rodriguez-Antolin A, Alekseev BY, et al. Abiraterone plus Prednisone in Metastatic, Castration-Sensitive Prostate Cancer. N Engl J Med. 2017;377(4):352-60.

34. de Bono JS, Oudard S, Ozguroglu M, Hansen S, Machiels JP, Kocak I, et al. Prednisone plus cabazitaxel or mitoxantrone for metastatic castration-resistant prostate cancer progressing after docetaxel treatment: a randomised open-label trial. Lancet. 2010;376(9747):1147-54.

35. Kluetz PG, Figg WD, Dahut WL. Angiogenesis inhibitors in the treatment of prostate cancer. Expert Opin Pharmacother. 2010;11(2):233-47.

36. Mulders PF, De Santis M, Powles T, Fizazi K. Targeted treatment of metastatic castration-resistant prostate cancer with sipuleucel-T immunotherapy. Cancer Immunol Immunother. 2015;64(6):655-63.

37. Schweizer MT, Drake CG. Immunotherapy for prostate cancer: recent developments and future challenges. Cancer Metastasis Rev. 2014;33(2-3):641-55.

38. Hwang C, Heath El. Angiogenesis inhibitors in the treatment of prostate cancer. J Hematol Oncol. 2010;3:26.

39. Stankova K, Brown JS, Dalton WS, Gatenby RA. Optimizing Cancer Treatment Using Game Theory: A Review. JAMA Oncol. 2018.

\section{Figures}
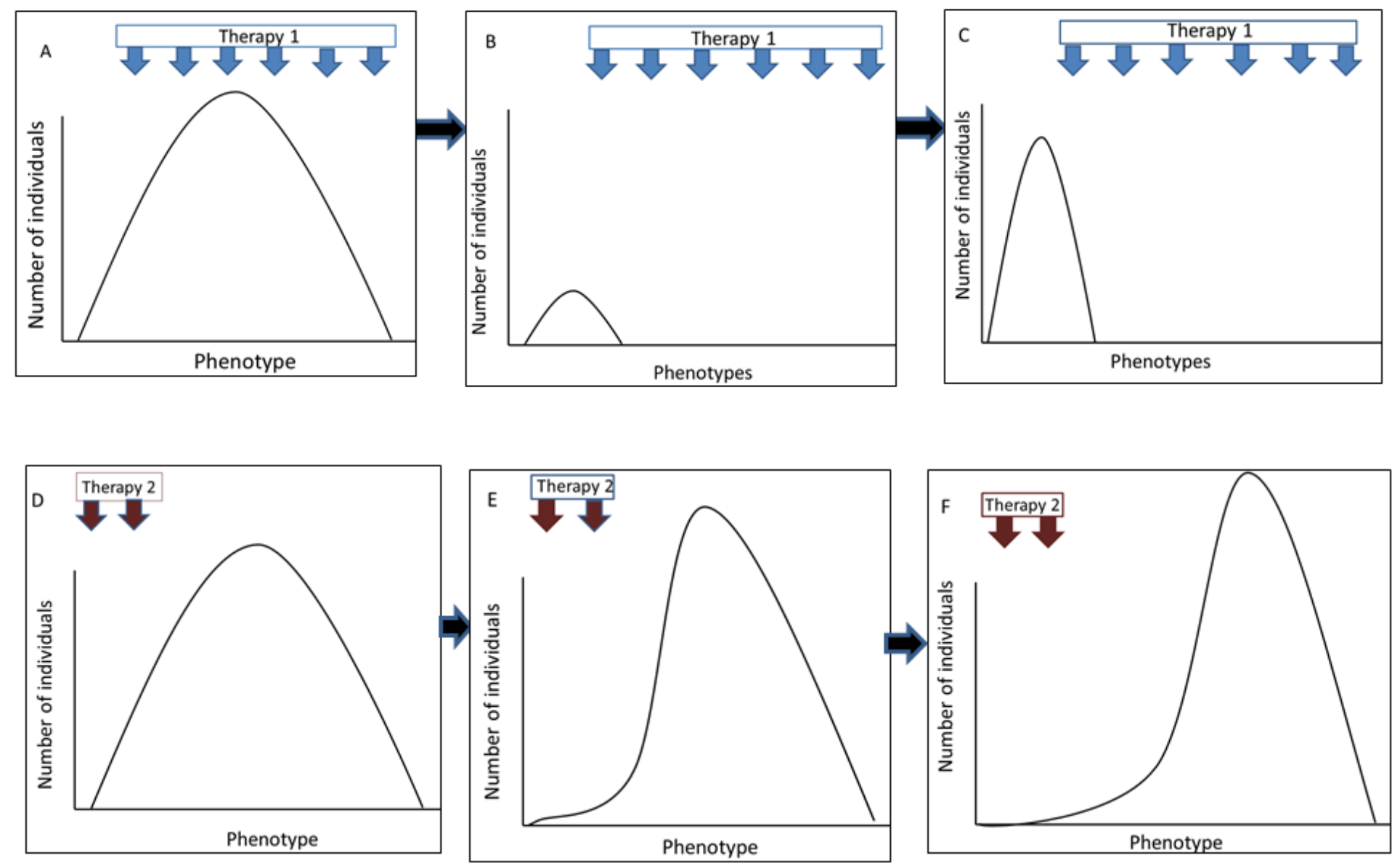
Figure 1. Conventional systemic therapy for large heterogeneous cancer population. Panel A demonstrates a heterogeneous population treated with some treatment strategy (Therapy 1). The tumor response is excellent leaving behind a small resistant population which is not clinically detectable but is also not affected by ongoing therapy. Eventually, this tumor population increases and becomes clinically apparent as progression. In this scenario, Therapy 1 is approved for treatment of this cancer but standard treatment strategy of maximum dose density until progression, eventually results in treatment failure and progression. Panel $D$ shows treatment with an alternative therapy. This treatment is lethal to only a small fraction of the tumor population that is not detectable by clinical imaging and tumor continues to increase in size. Thus, Treatment 2 is considered ineffective for treating this cancer type and is not considered a standard of care therapy.
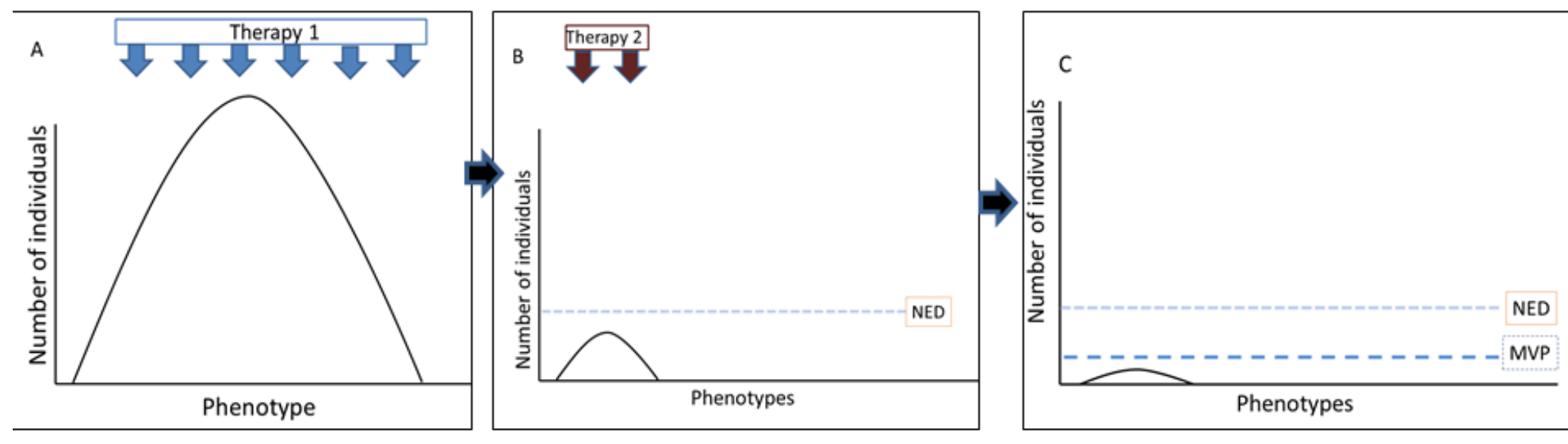

Figure 2. Imposing extinction dynamics on heterogeneous cancer populations. Panel A shows the same tumor populations and Therapy 1 as Figure 1. Here, Therapy 1 is discontinued once the patient is NED. Panel B show Therapy 2 administered immediately after Therapy 1 with the goal of reducing this small resistant population below the threshold of a Minimum Viable Population (MVP) (Panel C). Here, Therapy 2, although ineffective in treating the original tumor (Figure 1), can reduce a smaller, more homogenous population to below its MVP. In the context of background extinction dynamics, Therapy 1 is an effective first strike and has reduced both the size and heterogeneity of the cancer population. This small population is vulnerable to small perturbations which can result in extinction. 